\title{
Regular and frequent feedback of specific clinical criteria delivers a sustained improvement in the management of diabetic ketoacidosis 滥
}

\author{
Authors: Punith Kempegowda, ${ }^{\mathrm{A}}$ Ben Coombs, ${ }^{\mathrm{B}}$ Peter Nightingale, ${ }^{\mathrm{C}}$ Joht Singh Chandan, ${ }^{\mathrm{D}}$ Jaffar Al-Sheikhli, ${ }^{\mathrm{E}}$ \\ Bhavana Shyamanur, ${ }^{\mathrm{D}}$ Kasun Theivendran, ${ }^{\mathrm{D}}$ Anitha Vijayan Melapatte, ${ }^{\mathrm{F}}$ Umesh Salanke, ${ }^{\mathrm{G}}$ Mohammed $A{ }^{\mathrm{B} b e r},{ }^{\mathrm{G}}$ \\ Sandip Ghosh ${ }^{\mathrm{G}}$ and Parth Narendran ${ }^{\mathrm{H}}$
}

\begin{abstract}
Efficient management of diabetic ketoacidosis (DKA) improves outcomes and reduces length of stay. While clinical audit improves the management of DKA, significant and sustained improvement is often difficult to achieve. We aimed to improve the management of DKA in our trust through the implementation of quality improvement methodology. Five specific targets (primary drivers: fluid prescription, fixed rate intravenous insulin infusion, glucose measurement, ketone measurement and specialist referral) were selected following a baseline audit. Interventions (secondary drivers) were developed to improve these targets and included monthly feedback to departments of emergency medicine, acute medicine, and diabetes. Following our intervention, the mean average duration of DKA reduced from $\mathbf{2 2 . 0}$ hours to $\mathbf{1 0 . 2}$ hours. We demonstrate that regular audit cycles with interventions introduced through the plan-do-study-act model is an effective way to improve the management of DKA.
\end{abstract}

KEYWORDS: Causes, diabetic ketoacidosis, duration till resolution, length of stay, quality improvement

\footnotetext{
Authors: ${ }^{\text {A }}$ specialist registrar in diabetes, endocrinology and general internal medicine, Health Education West Midlands and honorary research fellow, Institute of Metabolism and Systems Research,

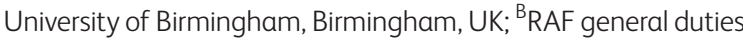
medical officer, Royal Centre for Defence Medicine, University Hospitals Birmingham NHS Foundation Trust, Birmingham, UK; ${ }^{{ }^{C}}$ statistician, Institute of Translational Medicine, University Hospitals Birmingham NHS Foundation Trust, Birmingham, UK; ' foundation year doctor, University Hospitals Birmingham NHS Foundation Trust, Birmingham, UK; ${ }^{\mathrm{E}}$ core medical trainee, University Hospitals Birmingham NHS Foundation Trust, Birmingham, UK; F senior data management developer - health informatics, University Hospitals Birmingham NHS Foundation Trust, Birmingham, UK; ${ }^{G}$ consultant, University Hospitals Birmingham NHS Foundation Trust, Birmingham, UK; ${ }^{H}$ reader and honorary consultant, University Hospitals Birmingham NHS Foundation Trust and Institute of Metabolism and Systems Research, University of Birmingham, Birmingham, UK
}

\section{Introduction}

Diabetic ketoacidosis (DKA) is a complex metabolic derangement defined by the presence of hyperglycaemia (blood glucose $>11 \mathrm{mmol} / \mathrm{L}$ ), acidosis $(\mathrm{pH}<7.3$ or bicarbonate $<15 \mathrm{mmol} / \mathrm{L}$ ) and ketonaemia (serum ketones $>3 \mathrm{mmol} / \mathrm{L}){ }^{1}{ }^{1}$ The 2016 National Diabetes Inpatient Audit identified that $6.7 \%$ of inpatients have type 1 diabetes in England. ${ }^{2} 45.9 \%$ patients with type 1 diabetes were admitted with DKA in $2015 .^{2}$ Although overall mortality rates of patients who present with DKA have improved over time, recent studies have reported that DKA continues to be a major problem, especially among young adults. ${ }^{3-6}$

The Joint British Diabetes Societies (JBDS) for Inpatient Care Group has produced guidance (2010, subsequently revised in 2013), standardising the management of DKA. This is supported by various professional organisations and patient groups (Diabetes UK, Association of British Clinical Diabetologists, British Society of Paediatric Endocrinology and Diabetes, Northern Irish Diabetologists, Society of Acute Medicine, Welsh Endocrine and Diabetes Society, and Scottish Diabetes Group), and guidelines for a good standard of care have been defined. ${ }^{1}$ While audits against these guidelines have been conducted at our institution to assess the quality of care for DKA, a sustained improvement in care has been difficult to establish. Contributing factors to this difficulty may include complex audit cycles, accessibility of medical records and the transitory rotations of junior doctors.

\section{Hypothesis and aims}

We hypothesised that implementing a quality improvement methodology for a limited number of important clinical criteria and frequent feedback to front-line staff would improve the implementation and adherence to the JBDS guidelines for DKA management. Our aim was to reduce the total duration of DKA by $50 \%$ in a sustainable manner. In order to achieve this, we identified five factors for improvement (primary drivers). We identified another five areas of intervention (secondary drivers) to achieve the improvement in primary drivers. 


\section{Methods}

The study was undertaken at University Hospitals Birmingham NHS Foundation Trust - a large tertiary care foundation trust in the West Midlands, UK - from April 2014 to September 2016. It is one of the largest hospitals in Western Europe with over 100,000 admissions per year. The study was divided into five distinct periods: pre-intervention (April 2014 to September 2014), intervention (October 2014 to March 2015), early followup (April 2015 to September 2015), intermediate follow-up (October 2015 to March 2015) and late follow-up (April 2016 to September 2016). All patients diagnosed with DKA according to national guidelines during the study period were included in the study. Patients managed in intensive care units were excluded from the study to avoid one-to-one care bias. Data was obtained with the help of informatics and electronic health records. The study was registered as a service improvement exercise with the trust's clinical governance team and did not require formal ethical review.

We initially conducted an audit assessing DKA management by retrospective medical record analysis between April 2014 and September 2014. The results were presented to frontline staff involved in the management of DKA (emergency medicine, acute medicine and diabetes teams). Following discussion, five (primary drivers) were identified:

\section{Fluid replacement}

Typically, patients with DKA are $100 \mathrm{~mL} / \mathrm{kg}$ fluid deficient at the time of presentation. Fluid replacement is the most important initial management. Guidelines suggest at least four litres of fluid replacement in the first 12 hours $(1,000 \mathrm{~mL}$ in the first hour, $2,000 \mathrm{~mL}$ over the next four hours and a further 2,000 $\mathrm{mL}$ over the next eight hours). ${ }^{1}$

2 Fixed rate intravenous insulin infusion (FRIII) Insulin replacement will switch off lipolysis, the main driver of metabolic acidosis in DKA. ${ }^{6}$ Guidelines recommend weight-based FRIII (calculated as 0.1 units/kg body weight) rather than the traditional variable rate insulin infusion in order to accommodate insulin-resistant states associated with obesity or pregnancy. ${ }^{1}$

\section{Glucose measurement}

Glucose falls sharply with insulin infusion; therefore, hourly glucose measurement is required. Supplementary glucose infusion may be required at the latter stages of DKA management to provide substrate and avoid hypoglycaemia until ketone production is completely switched off.

\section{Ketone measurement}

Guidelines recommend hourly ketone measurement to assess the adequacy of insulin replacement and also assess for resolution. Further titration of insulin infusion to aim for a reduction in blood ketone levels by approximately $0.5 \mathrm{mmol} / \mathrm{L} /$ hour.

\section{Specialist referral}

Patients with DKA should be referred to the diabetes specialist team on admission to ensure necessary specialist input is provided at the earliest opportunity.

A $50 \%$ reduction in duration of DKA was considered the primary aim. We collected data for patient demographics, aetiology of the DKA and the aforementioned parameters during the first 12 hours following the diagnosis of DKA.

We adopted the plan-do-study-act (PDSA) method to address the primary outcome in this study. The PDSA cycle is a system

\section{Box 1. The plan-do-act-study cycle}

> Plan - plan the test or observation, including a plan for
collecting data
$>$ Do - try out the test on a small scale
$>$ Study - analyse the data and study the results
$>$ Act - refine the change, based on what was learned from the test.

where an idea is tested by trialling a change or intervention and assessing its impact (Box 1). ${ }^{7,8}$ The various interventions (secondary drivers) introduced as part of our study are summarised in Fig 1:

\section{Developing a real time audit tool}

Our first intervention was to develop a real-time audit tool to minimise the delay between data collection and dissemination of results. We used existing electronic observation charts, prescribing and referral requests to capture these events. These enabled monitoring of the timings of glucose and ketone measurements, prescribing of fluids and insulin, and specialist referral requests

\section{Automatic referral to specialist team}

The electronic prescribing system was configured to generate an automatic referral to the diabetes team, triggered by FRIII prescription. Although the latter duplicated most of the referrals from DKA admission, it ensured none of the DKA admissions were missed from specialist review.

3 Electronic surveillance of blood gas results Point-of-care blood gas analyser reports were not originally incorporated in our electronic observation. Surveillance was improved by incorporating them into the electronic system for clinical monitoring. This allowed the DKA to be electronically monitored, and the timing of resolution based on the $\mathrm{pH}$, blood glucose and ketone measurement to be ascertained accurately.

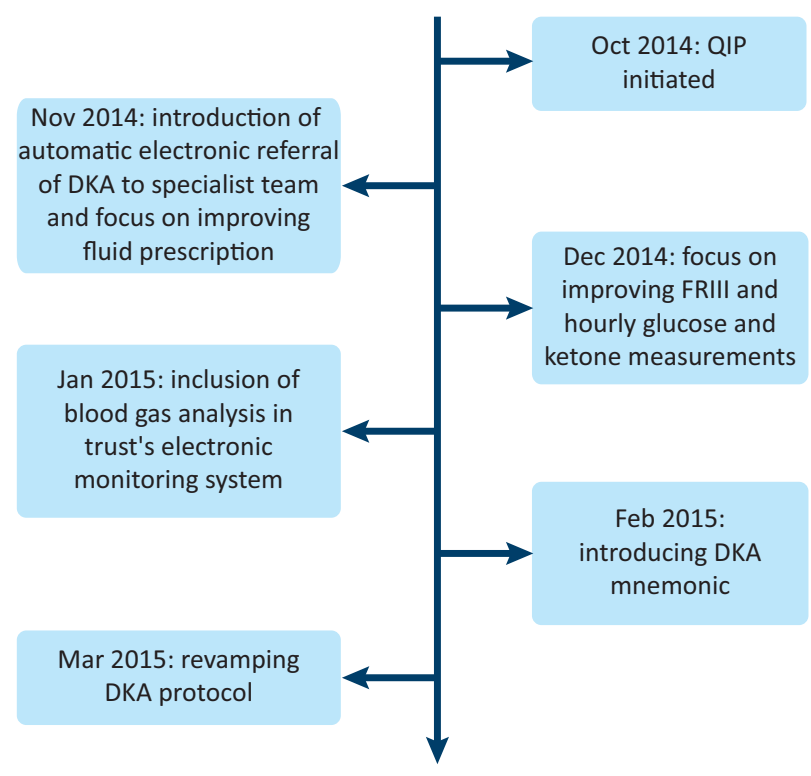

Fig 1. Timeline of interventions introduced during the study. DKA= diabetic ketoacidosis; FRIII = fixed rate intravenous insulin infusion; QIP = quality improvement project. 


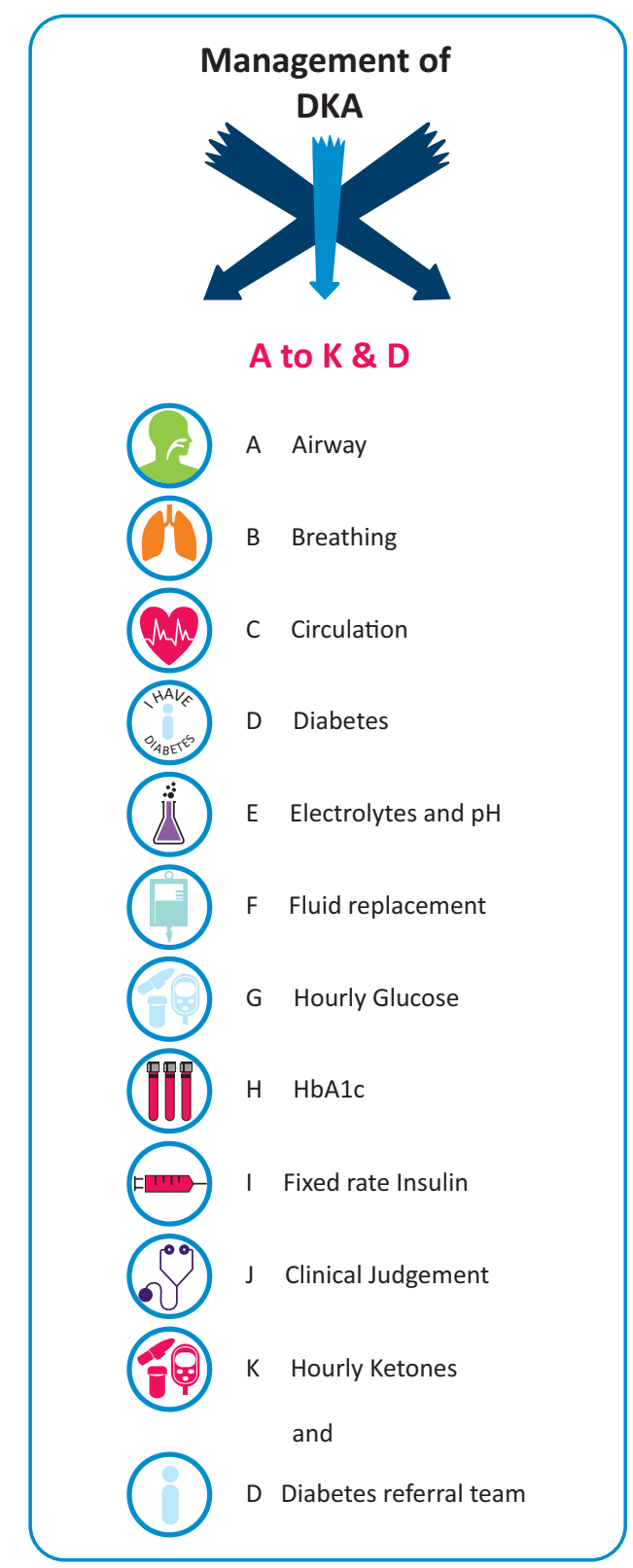

Fig 2. Mnemonic of the University Hospitals Birmingham NHS Foundation Trust's guidelines for management of diabetic ketoacidosis. This was used to improve adherence to the guidelines. DKA = diabetic ketoacidosis; $\mathrm{HbA1C}=$ glycated haemoglobin.

\section{Monthly feedback}

The results of monthly audits of the five primary drivers were presented to the front-line teams - departments of emergency medicine, acute medicine and diabetes via their clinical leads.

\section{Education and redesigning the trust guideline}

Feedback from junior doctors suggested that the current hospital guideline for management of DKA was difficult to follow and had 'too many words'. The guideline was therefore redesigned with an easy-to-remember mnemonic (Fig 2). The newly designed guideline was accessible through the trust's intranet. Printed versions of the guidelines were posted in emergency medicine and acute medical units where most of the DKA patients are admitted.

\section{Statistical analysis}

The data were analysed using SPSS version 21 (IBM Corp) and Prism 6.0 (Graphpad Inc). DKA duration was log transformed for analysis to obtain a variable that is approximately normally distributed and presented in geometric means and associated $95 \%$ confidence intervals $(\mathrm{CI})$. The remaining data are presented as median \pm interquartile range (IQR). Univariate analyses were performed using one-way analysis of variance, Kruskal-Wallis or Jonckheere-Terpstra tests for continuous and chi-square test or Kendall's tau-b for categorical variables. The variation of the clinical criteria chosen for the study was measured as the deviation from the national guideline's recommended value.

\section{Results}

A total of 259 episodes of DKA were included in the project. The baseline characteristics of the study population between the five groups are described in Table 1. There was no statistically significant difference in age distribution between the five groups. Poor compliance with treatment for diabetes and inter-current illness were the most common causes of DKA in our study. The total duration of DKA was reduced significantly following our intervention $(\mathrm{p}<0.001)$ (Fig 3). DKA duration (geometric mean and $95 \% \mathrm{CI}$ ) in the five groups were 22.0 (17.7-27.5) hours, 18.3 (15.2-22.0) hours, 12.0 (9.8-14.8) hours, 7.4 (6.4-8.7) hours and $10.2(8.2-12.7)$ hours, respectively. However, we did not notice a significant difference in the length of stay. The respective lengths of stay in the five groups were 75 (28-223) hours, 69 (41-179) hours, 87 (44-190), 71 (42-328) hours and 75 (48-149) hours. There was a significant reduction in the dispersion of values for FRIII. There was an increased proportion of values within $20 \%$ of the guideline value (67\% at pre-intervention to $92 \%$ in the late follow-up period $(\mathrm{p}=0.002)$ ). A similar trend was noted for hourly glucose measurements (the proportion of patients within $20 \%$ variation of the guideline increased from $33 \%$ to $47 \%$ ); however, this was not statistically significant $(\mathrm{p}=0.06)$. There was no significant change in the variation of fluid prescription and ketone measurements (Fig 4). All patients were referred to the diabetes team upon the diagnosis of DKA during the postintervention period.

\section{Discussion}

In this study, we show that auditing DKA management against five simple criteria and feeding the results back to front-line staff, along with systems enhancement for early identification and monitoring by a specialist team, reduces DKA duration in a sustained manner. We attribute this to a PDSA model of quality improvement.

The usual pattern of DKA management at our trust involves admission into the acute medical unit, through the emergency department, where they are managed until resolution. The patients' glucose and ketones are monitored hourly and necessary changes in insulin infusion and fluid replacement are made as per the trust's guidelines. Following resolution, patients are either discharged from the acute medical unit, if they have already been reviewed by the diabetes team, 
Table 1. Age, gender, aetiology and rates of new onset diabetes and specialist referral

\begin{tabular}{|c|c|c|c|c|c|}
\hline Group & $\begin{array}{l}\text { Pre-intervention } \\
(n=52)\end{array}$ & $\begin{array}{l}\text { Intervention } \\
(n=52)\end{array}$ & $\begin{array}{l}\text { Post-intervention } \\
(n=55)\end{array}$ & $\begin{array}{l}\text { Intermediate } \\
\text { follow-up }(n=64)\end{array}$ & $\begin{array}{l}\text { Late follow-up } \\
(n=36)\end{array}$ \\
\hline Median age, years (IQR) & $28(20-51)$ & $38(23-47)$ & $32(21-56)$ & $32(20-62)$ & $29(20-49)$ \\
\hline Male gender, $\mathrm{n}(\%)$ & $22(42.3)$ & $33(63.5)$ & $30(54.5)$ & $26(40.6)$ & $10(27.8)$ \\
\hline \multicolumn{6}{|l|}{ Aetiology, n(\%) } \\
\hline Alcohol-related & $1(1.9)$ & $5(9.6)$ & $2(3.6)$ & $1(1.6)$ & $2(5.6)$ \\
\hline Inter-current illness & $9(17.3)$ & $19(36.5)$ & $11(20.0)$ & $12(18.8)$ & $12(33.3)$ \\
\hline Poor compliance & $14(26.9)$ & $14(26.9)$ & $29(52.7)$ & $18(28.1)$ & $8(22.2)$ \\
\hline Sepsis & $6(11.5)$ & $2(3.8)$ & $2(3.6)$ & $9(14.1)$ & $3(8.3)$ \\
\hline Surgical & $8(15.4)$ & $5(9.6)$ & $2(3.6)$ & $7(10.9)$ & 0 \\
\hline New diagnosis of type 1 diabetes & $7(13.5)$ & $2(3.8)$ & $6(10.9)$ & $4(6.3)$ & $2(5.6)$ \\
\hline Unclear & $7(13.5)$ & $5(9.6)$ & $3(5.5)$ & $13(20.3)$ & $9(25.0)$ \\
\hline Referral to diabetes team & $48(92.3)$ & $37(71.2)$ & $55(100)$ & $64(100)$ & $36(100)$ \\
\hline
\end{tabular}

or transferred to a general medical ward where they await further review from the diabetes team and a safe discharge. The average healthcare cost in our acute medical unit is $\mathfrak{E} 14.1$ per hour. Therefore, assuming a pragmatic model where healthcare delivery remained the same throughout the study, DKA management from diagnosis to resolution in the acute medical unit cost $\mathfrak{E} 22,338.50$ at the beginning of the study. This reduced to $\mathfrak{E} 6,489.50$ at the end of the study. However, length of stay was not significantly reduced. This is likely due to factors that influence discharge after resolution of DKA (for example

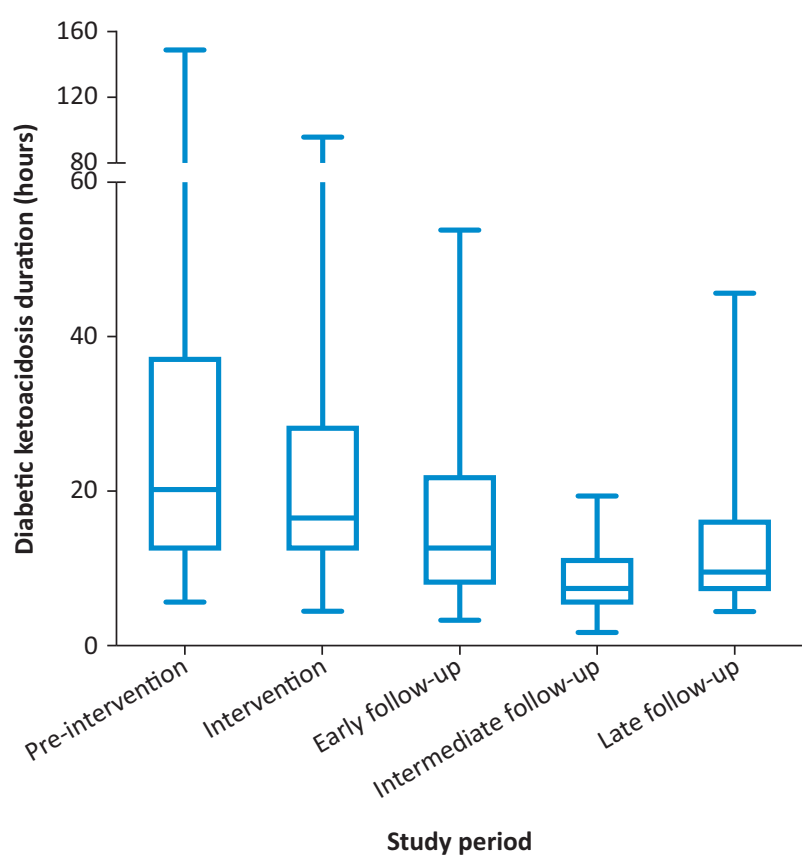

Fig 3. Diabetic ketoacidosis duration during five distinct periods. The geometric means (95\% CI) were 22.0 (17.7-27.5) hours pre-intervention, $18.3(15.2-22.0)$ hours during intervention, 12.0 (9.8-14.8) hours at early follow-up, 7.4 (6.4-8.7) hours at intermediate follow-up and 10.2 (8.2-12.7) hours at late follow-up. The total duration of diabetic ketoacidosis was reduced significantly following our intervention $(p<0.001)$. patient education, review of home support, dispensing of take home therapies). While the calculated savings are therefore hypothetical, the quicker resolution helps free up acute medical beds for other ill patients.

Delivering improvements in quality and patient safety remains a priority for the NHS. However, current methods lack a coherent, sustained and nationally replicable model. ${ }^{9}$ There is a need for multifaceted approaches that are iteratively able to adapt to local context and unforeseen difficulties. ${ }^{10}$ Furthermore, the NHS must effectively utilise 'organisational learning' skills through quality improvement to effectively sustain improvements in an ever changing healthcare system. ${ }^{11}$

We have demonstrated that the PDSA model of quality improvement, based around repeated assessment and intervention cycles, is an effective way to improve DKA management. Porter et al were able to demonstrate a significant and sustained improvement in diabetes management in their primary care in Western Australia by adopting the PDSA model. ${ }^{12}$ The model encourages organisational learning and communication through the development of dissemination methods. In comparison, a simple clinical audit may not have the required number of interventions and/or assessments to sustain improvement.

\section{Limitations}

Complications such as hypokalaemia and hypoglycaemia are associated with DKA management. ${ }^{13,14}$ These were not measured in this study and we were therefore unable to assess whether our intervention affected this outcome. We did not assess whether the shorter duration of DKA following the intervention affected DKA readmission rates. While DKA patients managed in the intensive care unit were excluded in the current study, we believe it was correct to do so. A recent survey of DKA management in the intensive care setting across East of England showed that majority of the centres do not follow JBDS guidelines. ${ }^{15}$ Also, these patients are managed with one-to-one nursing care with continuous monitoring, which may result in one-to-one care bias. Their inclusion would have skewed the results when compared with patients managed elsewhere with standard care. 


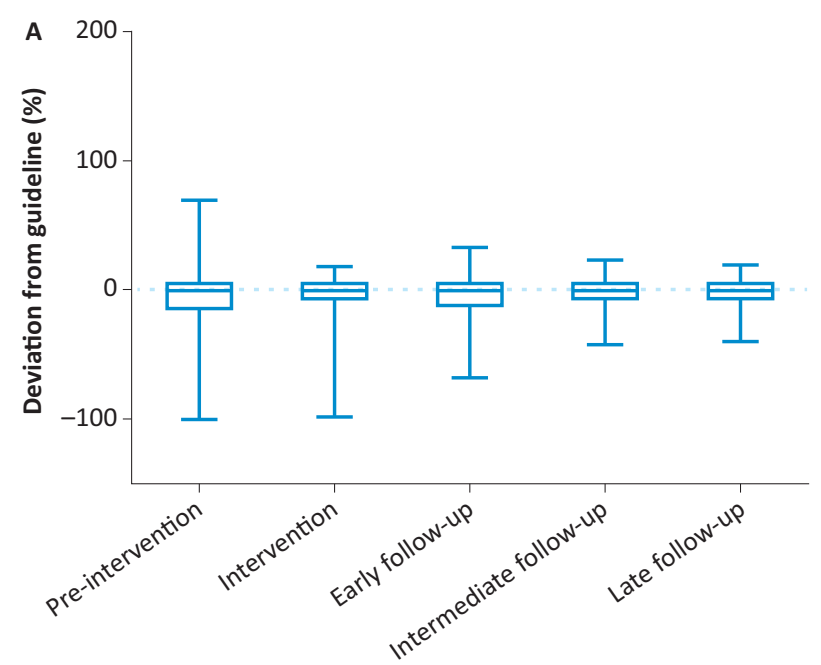

Study period

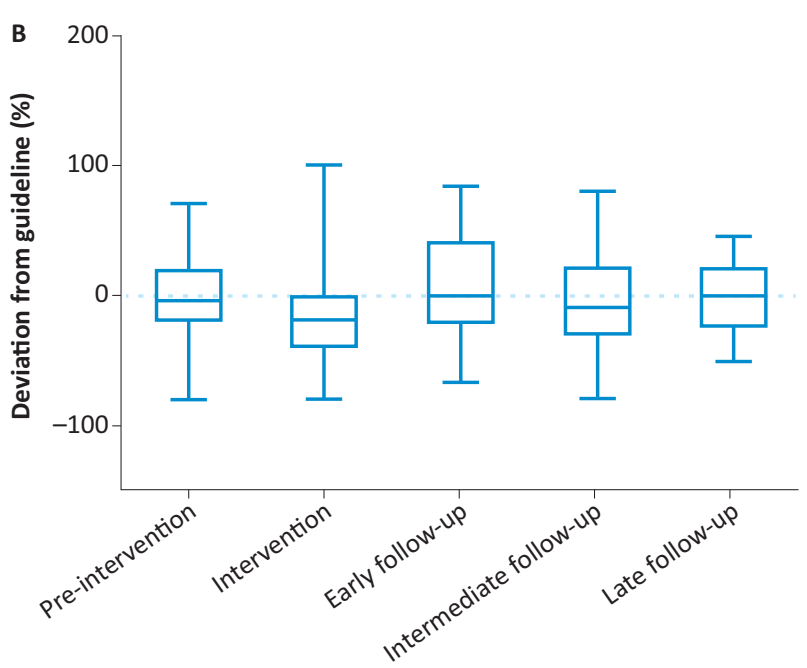

Study period

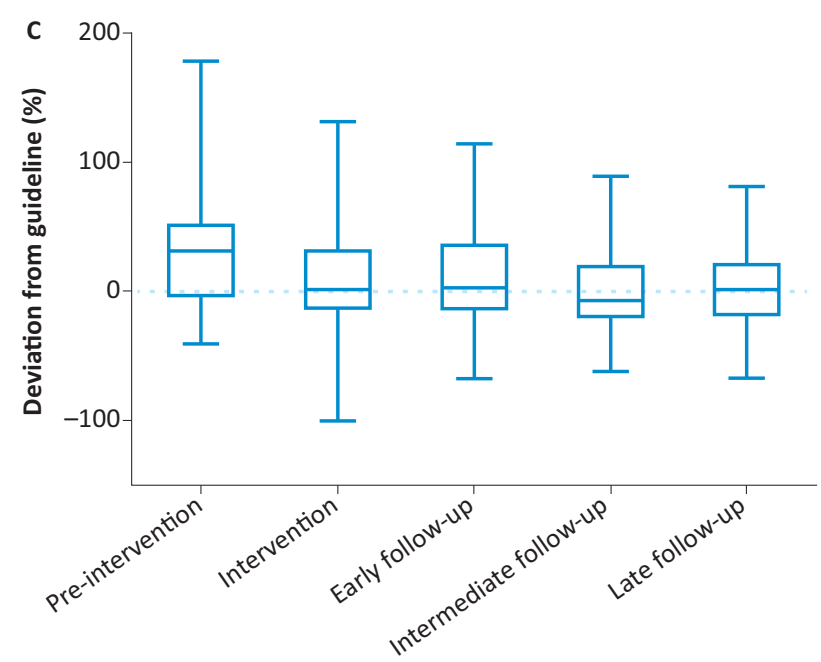

Study period

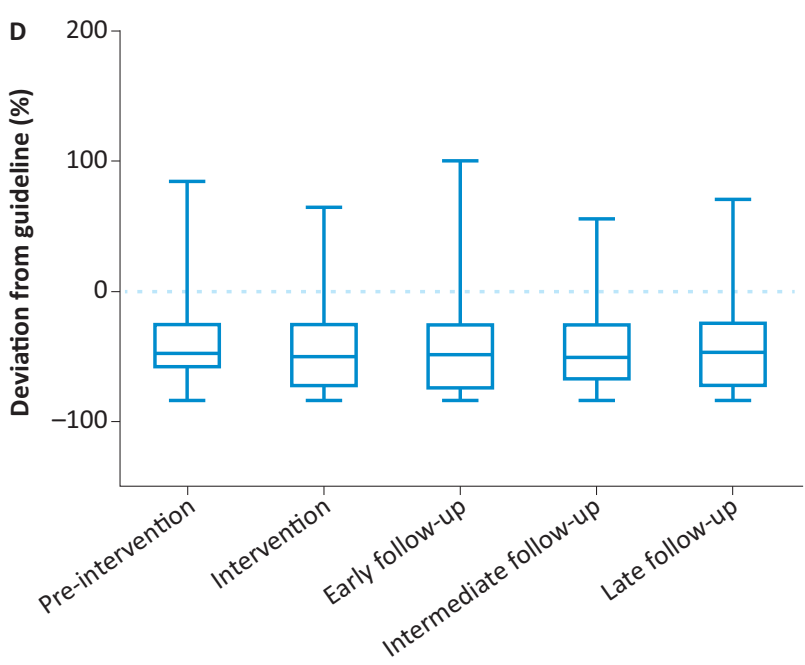

Study period

Fig 4. Deviation from guidance for four primary drivers for diabetic ketoacidosis management. A - fixed rate intravenous insulin infusion; B - fluids; $C$ - hourly glucose measurement; D - hourly ketone measurement. There was an increased proportion of values for fixed rate intravenous insulin infusion within the $20 \%$ of guideline value following our interventions during the study ( $67 \%$ at pre-intervention to $92 \%$ in the late follow-up period ( $\mathrm{p}=0.002)$ ). A similar trend was noted for hourly glucose measurements (the proportion of patients within $20 \%$ variation of the guideline increased from $33 \%$ to $47 \%$ ); however, this was not statistically significant ( $\mathrm{p}=0.06$ ). There was no significant change in the variation of fluid prescription and ketone measurements.

\section{Conclusions}

In our study, we were able to demonstrate a significant and sustained reduction in DKA. We attribute this to the PDSA model of quality improvement where we identified the primary drivers for change and developed secondary driver interventions to achieve the improvements. We propose that the primary drivers for improvement in resolution time of DKA be tested in other trusts to confirm reproducibility.

\section{Conflicts of interests}

The authors have no conflicts of interests to declare.

\section{Author contributions}

PK conceptualised, collected data, analysed data and wrote the first draft of the paper. BC, KT, JSC, JA-S and BS collected and analysed data, and contributed in designing and delivering various interventions during the study. US, MA, SG and PN supervised and contributed in refining the study and its interventions to have best impact. All authors critically reviewed and approved the final version of the paper.

\section{Acknowledgements}

We thank the staff of the emergency department, clinical decisions unit and the diabetes team for their help in this project. Our special gratitude to Mrs Theresa Smyth, Mrs Lesley Peters, Mrs Angela Phillips and the rest of the diabetes specialist nurses at our hospital for the help towards the success of the quality improvement project. We thank the health informatics and Mr Paolo Imbimbo's graphics team for their help in designing and introducing various electronic surveillance and educational intervention programmes. Finally, we would like to thank Mr Maurice Hakkak and Ms Leila Merchant for their inputs regarding financial and administrative aspects of diabetic ketoacidosis management. 


\section{References}

1 Savage MW, Dhatariya KK, Kilvert A et al. Joint British Diabetes Societies guideline for the management of diabetic ketoacidosis. Diabet Med 2011;28:508-15.

2 Health and Social Care Information Centre. National Diabetes Inpatient Audit 2015. National report. Leeds: HSCIC, 2016.

3 Abdulrahman GO, Amphlett B, Okosieme OE. Trends in hospital admissions with diabetic ketoacidosis in Wales, 1999-2010. Diabetes Res Clin Pract 2013;100:e7-10.

4 Gregg EW, Li Y, Wang J et al. Changes in diabetes-related complications in the United States, 1990-2010. N Engl J Med 2014;370:1514-23.

5 Jervis A, Champion S, Figg G et al. Prevalence of diabetes ketoacidosis rises and still no strict treatment adherence. Curr Diabetes Rev 2013;9:54-61.

6 Maahs DM, Hermann JM, Holman N et al. Rates of diabetic ketoacidosis: international comparison with 49,859 pediatric patients with type 1 diabetes from England, Wales, the US, Austria, and Germany. Diabetes Care 2015;38:1876-82.

7 Langley GJ, Moen RD, Nolan KM et al. The improvement guide: a practical approach to enhancing organizational performance, 2nd edn. San Francisco: Jossey-Bass,2009:490.

8 Gillam S, Siriwardena AN. Frameworks for improvement: clinical audit, the plan-do-study-act cycle and significant event audit. Qual Prim Care 2013;21:123-30.

9 Ham C, Berwick D, Dixon J. Improving quality in the English NHS: a strategy for action. London: King's Fund, 2016.
10 Taylor MJ, McNicholas C, Nicolay C et al. Systematic review of the application of the plan-do-study-act method to improve quality in healthcare. BMJ Qual Saf 2014;23:290-8.

11 Carroll JS, Edmondson AC. Leading organisational learning in health care. Qual Saf Health Care 2002;11:51-6.

12 Porter C, Greenfield C, Larson A, Gilles M. Improving GP diabetes management - A PDSA audit cycle in Western Australia. Aust Fam Physician 2009;38:939-44.

13 Dhatariya KK, Nunney I, Higgins K et al. National survey of the management of Diabetic Ketoacidosis (DKA) in the UK in 2014. Diabet Med 2016;33:252-60.

14 Varadarajan M, Patel M, Kakkar N et al. Are the results from the 2014 UK national survey on the management of diabetic ketoacidosis applicable to individual centres? Diabetes Res Clin Pract 2017;127:140-6.

15 Rudd B, Patel K, Levy N et al. A survey of the implementation of the NHS diabetes guidelines for management of diabetic ketoacidosis in the intensive care units of the East of England. J Intensive Care Soc 2013;14:60-4

Address for correspondence: Dr Punith Kempegowda,

Department of Diabetes and Endocrinology, Queen Elizabeth Hospital Birmingham, Mindelsohn Way, Birmingham B15 2GW, UK.

Email: p.kempegowda@nhs.net
Assessing trainees in the workplace An e-learning module for secondary care doctors

\author{
Three hours of CPD-approved interactive \\ learning covering: \\ $>$ feedback \\ > supervised learning events (SLES) \\ $>$ workplace-based assessments (WPBAs) \\ $>$ the role of the Annual Review of \\ Competence Progression (ARCP). \\ For more information please visit: \\ www.rcplondon.ac.uk/elearning
}

\section{Royal College of Physicians}

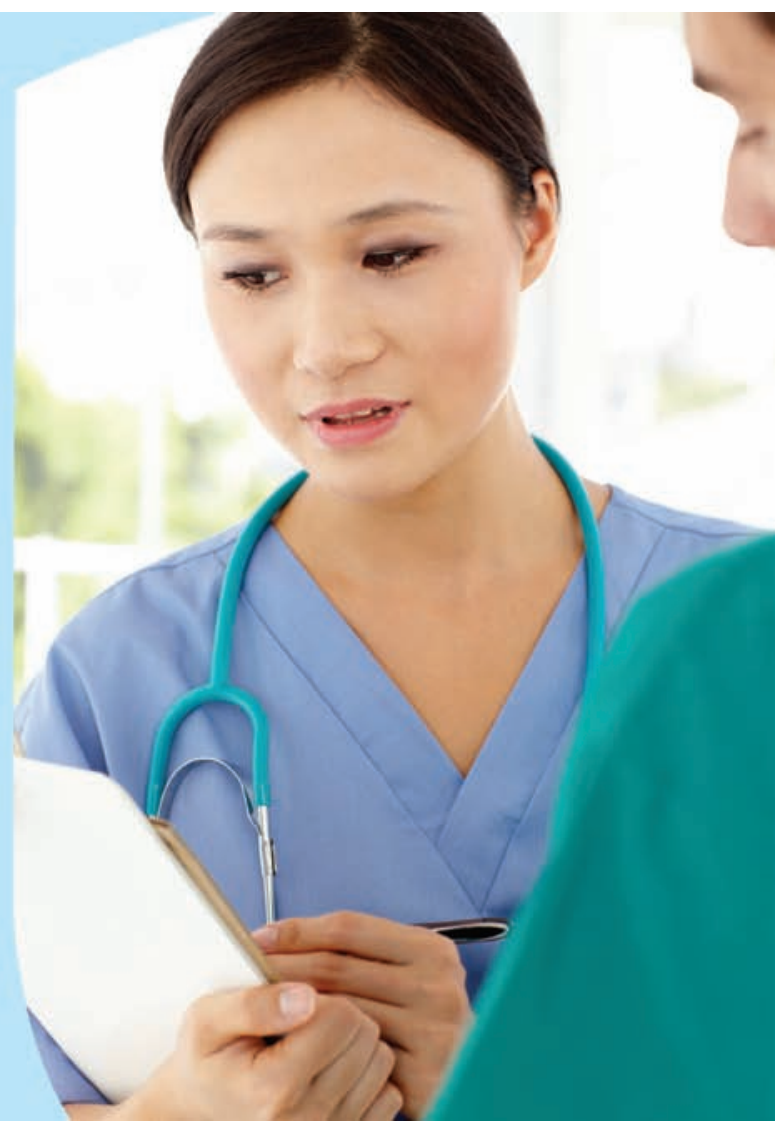

